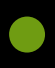 \\ hre a journal of mathematics
}

Zero forcing number, path cover number, and maximum nullity of cacti

Darren D. Row

mathematical sciences publishers 


\title{
Zero forcing number, path cover number, and maximum nullity of cacti
}

\author{
Darren D. Row \\ (Communicated by Chi-Kwong Li)
}

The zero forcing number of a graph is the minimum size of a zero forcing set. This parameter is useful in the minimum rank/maximum nullity problem, as it gives an upper bound to the maximum nullity. The path cover number of a graph is the minimum size of a path cover. Results for comparing the parameters are presented, with equality of zero forcing number and path cover number shown for all cacti and equality of zero forcing number and maximum nullity for a subset of cacti. (A cactus is a graph where each edge is in at most one cycle.)

\section{Introduction}

Throughout this paper, a graph $G=\left(V_{G}, E_{G}\right)$ will mean a simple (no loops, no multiple edges) undirected graph. We will assume a finite and non-empty vertex set $V_{G}$. The edge set $E_{G}$ consists of two-element subsets of vertices. If $\{x, y\} \in E_{G}$, we say $x$ and $y$ are neighbors or $x$ and $y$ are adjacent, and write $x \sim y$.

The zero forcing number of a graph was introduced in [AIM 2008] and the related terminology was developed in [Barioli et al. 2009], [Barioli et al. 2010], and [Hogben 2010]. Referring to it as the graph infection number, physicists have used this parameter in studying quantum systems control [Burgarth and Giovannetti 2007; Burgarth and Maruyama 2009; Severini 2008]. Consider a black and white vertex coloring of a graph $G$. From the initial coloring, vertices change color according to the color-change rule: If $v$ is the only white neighbor of a black vertex $u$, then change the color of $v$ to black. Applying the color-change rule to $u$ to change the color of $v$, we say $u$ forces $v$ and write $u \rightarrow v$. Given an initial coloring of $G$, the derived set is the set of vertices colored black after the colorchange rule is applied until no more changes are possible. If the set $Z$ of vertices initially colored black has derived set that is all the vertices of $G$, we say $Z$ is a zero forcing set for $G$. A zero forcing set with the minimum number of vertices is called an optimal zero forcing set, and this minimum size of a zero forcing set for a graph $G$ is the zero forcing number of the graph, denoted $\mathrm{Z}(G)$.

MSC2010: primary 05C50; secondary 15A03.

Keywords: zero forcing number, path cover number, maximum nullity, cut-edge, cut-vertex, cactus. 
The path cover number $\mathrm{P}(G)$ of a graph $G$ is the smallest positive integer $m$ such that there are $m$ vertex-disjoint induced paths in $G$ such that every vertex of $G$ is a vertex of one of the paths.

An association between graphs and matrices is made in the following way. Denote by $S_{n}(\mathbb{R})$ the set of $n \times n$ real symmetric matrices. The graph of $A \in S_{n}(\mathbb{R})$, denoted $\mathscr{G}(A)$, is the graph with vertices $\{1, \ldots, n\}$ and edges $\left\{\{i, j\}: a_{i j} \neq 0,1 \leq\right.$ $i<j \leq n\}$. Given a graph $G$, the set of symmetric matrices described by $G$ is $\mathscr{S}(G)=\left\{A \in S_{n}(\mathbb{R}): \mathscr{G}(A)=G\right\}$. The minimum rank of $G$ is $\operatorname{mr}(G)=\min \{\operatorname{rank} A$ : $A \in \mathscr{S}(G)\}$ and the maximum nullity of $G$ is $\mathrm{M}(G)=\max \{$ null $A: A \in \mathscr{Y}(G)\}$. Clearly $\operatorname{mr}(G)+\mathrm{M}(G)=|G|$, where the order $|G|$ is the number of vertices in $G$. Because of this relationship, finding the value of one of these two parameters for a graph is equivalent to finding the value for both.

Following are theorems relating the zero forcing number to path cover number and maximum nullity of a graph. These bounds will be used in later results.

Theorem 1.1 [Hogben 2010]. For any graph $G, \mathrm{P}(G) \leq \mathrm{Z}(G)$.

Theorem 1.2 [AIM 2008]. For any graph $G, \mathrm{M}(G) \leq \mathrm{Z}(G)$.

It is well known that if $G$ is a tree then $\mathrm{P}(G)=\mathrm{Z}(G)$ [AIM 2008] and $\mathrm{P}(G)=$ $\mathrm{M}(G)$ [Johnson and Duarte 1999], so the three parameters are equal.

In this paper, we compare the graph parameters $\mathrm{Z}(G), \mathrm{P}(G)$, and $\mathrm{M}(G)$. In Section 2, we present the effect on the parameters after the deletion of a single vertex or the deletion of a single edge. These (mostly known) results will be utilized in later sections. Results of similar type for each of the graph parameters are presented in a unified format to emphasize the relationship to each other. The main result of Section 3 is equality of zero forcing number and path cover number for cacti, where a cactus is a graph where each edge is in at most one cycle. In Section 4, we prove zero forcing number is equal to maximum nullity for a restricted family of cacti. Section 5 summarizes our results and suggests further research.

Additional properties and some notation. Here we present additional terminology, notation, and theorems that will be used. For a given zero forcing set $Z$, a chronological list of forces is a listing of the forces used to construct the derived set in the order they are performed. A forcing chain for a chronological list of forces is a sequence of vertices $\left(v_{1}, v_{2}, \ldots, v_{k}\right)$ such that for $i=1, \ldots, k-1$, $v_{i} \rightarrow v_{i+1}$, and a maximal forcing chain is a forcing chain that is not a proper subsequence of any other forcing chain. The collection of maximal forcing chains for a chronological list of forces is called the chain set of the chronological list of forces, and an optimal chain set is a chain set from a chronological list of forces of an optimal zero forcing set. When a chain set contains a chain consisting of a single vertex, we say that the chain set contains the vertex as a singleton. For a 
zero forcing set $Z$, a reversal of $Z$ is the set of vertices which are last in the forcing chains in the chain set of some chronological list of forces [Barioli et al. 2010].

Theorem 1.3 [Barioli et al. 2010]. If $Z$ is a zero forcing set of $G$ then so is any reversal of $Z$.

Observation 1.4. If $Z^{\prime}$ is a reversal of $Z$, then $\left|Z^{\prime}\right|=|Z|$. In particular, if $Z$ is an optimal zero forcing set, then a reversal $Z^{\prime}$ of $Z$ is also an optimal zero forcing set.

A vertex $v$ is called terminal if it is the endpoint of a path in some minimum path cover. It is called doubly terminal if it is in a path by itself in some minimum path cover, and is called simply terminal if it is terminal but not doubly terminal.

For a graph $G=\left(V_{G}, E_{G}\right)$ and $W \subseteq V_{G}$, the induced subgraph $G[W]$ is the graph with vertex set $W$ and edge set $\left\{\{v, w\} \in E_{G}: v, w \in W\right\}$. The subgraph induced by $\bar{W}=V_{G} \backslash W$ will be denoted by $G-W$, or in the case $W$ is a single vertex $\{v\}$, by $G-v$. For $e \in E_{G}$, the subgraph $\left(V_{G}, E_{G} \backslash\{e\}\right)$ will be denoted by $G-e$.

A graph is called connected if any two vertices are linked by a path. If a graph is not connected, we say it is disconnected. The maximal connected subgraphs of a graph are called the components of the graph. If the graph $G-v$ has more connected components than $G$, then $v$ is called a cut-vertex of $G$. Similarly, a cut-edge of a graph is one such that its deletion increases the number of connected components.

\section{Edge spread and vertex spread}

We present a number of (mostly known) results which will be used in later sections. They are grouped and formatted in such a way as to emphasize commonality between the types of results for the different parameters.

Edge spread. In this subsection, we consider the effects on zero forcing number, path cover number, and maximum nullity when deleting a single edge from a graph. For a graph $G$ and an edge $e$ of $G$, the rank edge spread of $e$ in $G$ is $\mathrm{r}_{e}(G)=$ $\operatorname{mr}(G)-\operatorname{mr}(G-e)$, the null edge spread of $e$ in $G$ is $\mathrm{n}_{e}(G)=\mathrm{M}(G)-\mathrm{M}(G-e)$, and the zero edge spread of $e$ in $G$ is $\mathrm{z}_{e}(G)=\mathrm{Z}(G)-\mathrm{Z}(G-e)$ [Edholm et al. 2010]. Here we make an analogous definition concerning change in path cover number when deleting an edge.

Definition 2.1. The path edge spread of $e$ in $G$ is $\mathrm{p}_{e}(G)=\mathrm{P}(G)-\mathrm{P}(G-e)$.

First we present the bounds on the zero edge spread and path edge spread and attempt to characterize edges with a given edge spread value.

Theorem 2.2 [Edholm et al. 2010]. For every graph $G$ and every edge $e=\{v, w\}$ of $G,-1 \leq \mathrm{z}_{e}(G) \leq 1$. If $\mathrm{z}_{e}(G)=1$, then there exists an optimal chain set such that e is not an edge in any chain. 
Theorem 2.3. For every graph $G$ and every edge $e=\{v, w\}$ of $G,-1 \leq \mathrm{p}_{e}(G) \leq 1$. If $\mathrm{p}_{e}(G)=1$, then there exists a minimum path cover such that $v$ and $w$ are not in the same path.

Proof. Let $G$ be a graph and $e=\{v, w\}$ be an edge in $G$. Consider a minimum path cover of $G$. If $v$ and $w$ are not covered by the same path, then this path cover of $G$ is also a path cover of $G-e$. If $v$ and $w$ are covered by the same path in the path cover of $G$, then splitting the path into two paths will create a path cover of $G-e$. Either way, $\mathrm{P}(G-e) \leq \mathrm{P}(G)+1$ so $\mathrm{p}_{e}(G) \geq-1$.

Consider a minimum path cover of $G-e$. If $v$ and $w$ are not covered by the same path, then this path cover of $G-e$ is also a path cover of $G$ (observe that this case cannot occur if $\left.\mathrm{p}_{e}(G)=1\right)$. If $v$ and $w$ are covered by the same path in the path cover of $G-e$, there must be a vertex on the path between them. Let $x$ be the vertex that is between $v$ and $w$ on the path and adjacent to $v$. Split the path between $v$ and $x$. This is a path cover of $G$, but with one more than $\mathrm{P}(G-e)$ paths. In the case $\mathrm{p}_{e}(G)=1$, this is a minimum path cover of $G$ with $v$ and $w$ in different paths. Regardless of the path edge spread, $\mathrm{P}(G) \leq \mathrm{P}(G-e)+1$ so $\mathrm{p}_{e}(G) \leq 1$.

Theorem 2.4 [Edholm et al. 2010]. Let $e=\{v, w\}$ be an edge of $G$. If $\mathrm{z}_{e}(G)=-1$, then for every optimal zero forcing chain set of $G, e$ is an edge in a chain.

Theorem 2.5. Let $e=\{v, w\}$ be an edge of $G$. If $\mathrm{p}_{e}(G)=-1$, then for every minimum path cover of $G, v$ and $w$ are in the same path.

Proof. The contrapositive will be proved. Let $G$ be a graph and $e=\{v, w\}$ be an edge of $G$. Suppose there is a minimum path cover of $G$ in which $v$ and $w$ are not in the same path. This path cover of $G$ is also a path cover of $G-e$, so $\mathrm{P}(G-e) \leq \mathrm{P}(G)$. Hence $\mathrm{p}_{e}(G) \geq 0$.

Theorem 2.5 can be viewed as a partial converse to the second statement in Theorem 2.3. Here we provide an example showing that the converse of the second statement in Theorem 2.3 is not true. This example also shows the converse of the second statement in Theorem 2.2 is false.

Example 2.6. Let $G$ be this graph:

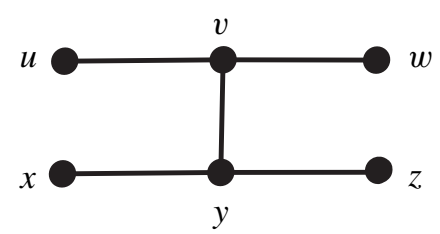

For $e=\{v, y\}$ we have $\mathrm{p}_{e}(G)=0$, but $v$ and $y$ are not in the same path in the minimum path cover. 
Although the bounds on $\mathrm{z}_{e}(G)$ and $\mathrm{p}_{e}(G)$ are the same, the parameters are not generally comparable, as can be seen in Examples 2.7 and 2.8 below. Null edge spread has the same bounds as well, and [Edholm et al. 2010] gives examples showing the incomparability of $\mathrm{z}_{e}(G)$ with $\mathrm{n}_{e}(G)$.

Example 2.7. Let $G$ be this graph:

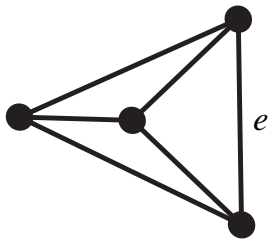

Here $\mathrm{Z}(G)=3$ and $\mathrm{Z}(G-e)=\mathrm{P}(G)=\mathrm{P}(G-e)=2$. Therefore, $\mathrm{z}_{e}(G)=1>$ $0=\mathrm{p}_{e}(G)$.

Example 2.8. Let $G$ be this graph:

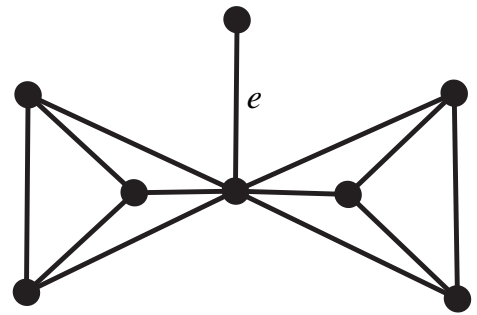

Here $\mathrm{Z}(G)=5, \mathrm{Z}(G-e)=6$, and $\mathrm{P}(G)=\mathrm{P}(G-e)=4$. Therefore, $\mathrm{z}_{e}(G)=$ $-1<0=\mathrm{p}_{e}(G)$.

Under the conditions of Observation 2.9 we can use one of parameters $\mathrm{z}_{e}(G)$ or $\mathrm{p}_{e}(G)$ to determine the other.

Observation 2.9. Let $G$ be a graph such that $\mathrm{P}(G)=\mathrm{Z}(G)$ and let $e$ be an edge of $G$. Then:

(1) $\mathrm{p}_{e}(G) \geq \mathrm{z}_{e}(G)$.

(2) If $\mathrm{z}_{e}(G)=1$, then $\mathrm{p}_{e}(G)=1$.

(3) If $\mathrm{p}_{e}(G)=-1$, then $\mathrm{z}_{e}(G)=-1$.

Next we consider edge spreads when the edge is a cut-edge.

Theorem 2.10 [Barioli et al. 2004]. Let $e=\left\{v_{1}, v_{2}\right\}$ be a cut-edge of a connected graph $G$. Let $G_{1}$ and $G_{2}$ be the connected components of $G-e$ with $v_{1} \in G_{1}$ and $v_{2} \in G_{2}$. Then

$$
\mathrm{r}_{e}(G)= \begin{cases}0 & \text { if } \max _{i=1,2}\left\{r_{v_{i}}\left(G_{i}\right)\right\}=2 \\ 1 & \text { otherwise }\end{cases}
$$


Corollary 2.11. Let $e=\left\{v_{1}, v_{2}\right\}$ be a cut-edge of a connected graph G. Let $G_{1}$ and $G_{2}$ be the connected components of $G-e$ with $v_{1} \in G_{1}$ and $v_{2} \in G_{2}$. Then

$$
\mathrm{n}_{e}(G)=\left\{\begin{aligned}
0 & \text { if } \min _{i=1,2}\left\{n_{v_{i}}\left(G_{i}\right)\right\}=-1 \\
-1 & \text { otherwise }
\end{aligned}\right.
$$

Proof. This follows from Theorem 2.10 and the fact that $\mathrm{r}_{e}(G)+\mathrm{n}_{e}(G)=0$ for any graph $G$ and any edge $e$ of $G$.

Theorem 2.12. Let $e=\left\{v_{1}, v_{2}\right\}$ be a cut-edge of a connected graph $G$. Let $G_{1}$ and $G_{2}$ be the connected components of $G-e$ with $v_{1} \in G_{1}$ and $v_{2} \in G_{2}$. Then

$$
\mathrm{z}_{e}(G)=\left\{\begin{aligned}
-1 & \text { if } v_{i} \text { is in an optimal zero forcing set in } G_{i} \text { for } i=1,2, \\
0 & \text { otherwise. }
\end{aligned}\right.
$$

Proof. Let $Z_{1}$ and $Z_{2}$ be optimal zero forcing sets for $G_{1}$ and $G_{2}$, respectively. Let $Z=Z_{1} \cup Z_{2}$. Color the vertices of $Z$ black and the remaining vertices white. Forces can be performed in $G_{1}$ until $v_{1}$ is black. Forces can be performed in $G_{2}$ until $v_{2}$ is black. Now the remaining forces can take place in $G_{1}$ and in $G_{2}$. Therefore $Z$ is a zero forcing set for $G$ and $\mathrm{Z}(G) \leq|Z|=\mathrm{Z}\left(G_{1}\right)+\mathrm{Z}\left(G_{2}\right)=\mathrm{Z}(G-e)$. Hence $\mathrm{z}_{e}(G) \leq 0$.

Suppose $v_{1}$ is an optimal zero forcing set $Z_{1}$ for $G_{1}$ and $v_{2}$ is in an optimal zero forcing set $Z_{2}$ in $G_{2}$. Let $Z_{1}^{\prime}$ be a reversal of $Z_{1}$. Then by Observation 1.4, $Z_{1}^{\prime}$ is an optimal zero forcing set for $G_{1}$ and there is a chronological list of forces in which $v_{1}$ does not perform a force (i.e., $v_{1}$ is last in the maximal forcing chain which contains it). Let $Z=Z_{1}^{\prime} \cup Z_{2} \backslash\left\{v_{2}\right\}$. Color the vertices of $Z$ black and the remaining vertices white. Forces can be performed in $G_{1}$ until all vertices of $G_{1}$ are black and $v_{1}$ has not performed a force. Now $v_{1}$ is black and $v_{2}$ is the only white neighbor of $v_{1}$, so $v_{1} \rightarrow v_{2}$. Now all the vertices of $Z_{2}$ are black and none has performed a force, so all other vertices of $G_{2}$ can be forced black. Therefore $\mathrm{Z}$ is a zero forcing set for $G$ and $\mathrm{Z}(G) \leq|Z|=\mathrm{Z}\left(G_{1}\right)+\mathrm{Z}\left(G_{2}\right)-1=\mathrm{Z}(G-e)-1$. Theorem 2.2 gives $\mathrm{z}_{e}(G) \geq-1$, so $\mathrm{z}_{e}(G)=-1$.

Suppose now that at least one of $v_{1}$ or $v_{2}$ is not in any optimal zero forcing set for the respective component. Without loss of generality, say $v_{1}$ is not in any optimal zero forcing set for $G_{1}$. Let $Z$ be an optimal zero forcing set for $G$ and consider the chronological list of forces. Examine the following cases.

Case 1: Suppose $v_{1} \rightarrow v_{2}$. Then $v_{1}$ cannot force any vertex of $G_{1}$. Since $v_{1}$ is not in any optimal zero forcing set for $G_{1}$, it is not at the end of a forcing chain for any optimal zero forcing set of $G_{1}$. Thus $v_{1}$ forcing $v_{2}$ requires $\left|Z \cap V_{G_{1}}\right| \geq Z\left(G_{1}\right)+1$. It must also be that $\left|Z \cap V_{G_{2}}\right| \geq Z\left(G_{2}\right)-1$. Then $Z(G)=|Z|=\left|Z \cap V_{G_{1}}\right|+\left|Z \cap V_{G_{2}}\right| \geq$ $\mathrm{Z}\left(G_{1}\right)+\mathrm{Z}\left(G_{2}\right)=\mathrm{Z}(G-e)$, so $\mathrm{z}_{e}(G) \geq 0$. 
Case 2: Suppose $v_{1} \nrightarrow \rightarrow v_{2}$. Then $\left|Z \cap V_{G_{2}}\right| \geq Z\left(G_{2}\right)$. Since $v_{1}$ is not in any optimal zero forcing set for $G_{1}$, it must be that $\left|Z \cap V_{G_{1}}\right| \geq Z\left(G_{1}\right)$. Then $\mathrm{Z}(G)=$ $|Z|=\left|Z \cap V_{G_{1}}\right|+\left|Z \cap V_{G_{2}}\right| \geq \mathrm{Z}\left(G_{1}\right)+\mathrm{Z}\left(G_{2}\right)=\mathrm{Z}(G-e)$, so $\mathrm{z}_{e}(G) \geq 0$.

Theorem 2.13 [Barioli et al. 2004]. Let $e=\left\{v_{1}, v_{2}\right\}$ be a cut-edge of a connected graph $G$. Let $G_{1}$ and $G_{2}$ be the connected components of $G-e$ with $v_{1} \in G_{1}$ and $v_{2} \in G_{2}$. Then

$$
\mathrm{p}_{e}(G)=\left\{\begin{aligned}
-1 & \text { if } v_{i} \text { is terminal in } G_{i} \text { for } i=1,2, \\
0 & \text { otherwise. }
\end{aligned}\right.
$$

The converse of Theorem 2.4 is open from [Edholm et al. 2010], and the converse of Theorem 2.5 is left open in this paper. We will show that the converses of these theorems are true for a cut-edge.

Theorem 2.14. Let $e=\{v, w\}$ be a cut-edge of $G$. If $e$ is an edge in a chain for every optimal zero forcing chain set of $G$, then $\mathrm{z}_{e}(G)=-1$.

Proof. The contrapositive will be proved. Suppose $\mathrm{z}_{e}(G) \neq-1$. By Theorem 2.12, $\mathrm{z}_{e}(G)=0$. Let $G_{1}$ and $G_{2}$ be the connected components of $G-e$ with $v \in G_{1}$ and $w \in G_{2}$. Let $Z_{1}$ and $Z_{2}$ be optimal zero forcing sets for $G_{1}$ and $G_{2}$, respectively. Let $Z=Z_{1} \cup Z_{2}$. Color the vertices of $Z$ black and the remaining vertices white. Forces can be performed in $G_{1}$ until $v$ is black. Forces can be performed in $G_{2}$ until $w$ is black. Now the remaining forces can take place in $G_{1}$ and in $G_{2}$. Therefore $Z$ is a zero forcing set for $G$ and $e=\{v, w\}$ is not an edge in any chain. Also, $|Z|=\mathrm{Z}\left(G_{1}\right)+\mathrm{Z}\left(G_{2}\right)=\mathrm{Z}(G-e)=\mathrm{Z}(G)-\mathrm{z}_{e}(G)=\mathrm{Z}(G)$, so $Z$ is an optimal zero forcing set for $G$.

Theorem 2.15. Let $e=\{v, w\}$ be a cut-edge of $G$. If $v$ and $w$ are in the same path for every minimum path cover of $G$, then $\mathrm{p}_{e}(G)=-1$.

Proof. The contrapositive will be proved. Suppose $\mathrm{p}_{e}(G) \neq-1$. By Theorem 2.13, $\mathrm{p}_{e}(G)=0$. Let $G_{1}$ and $G_{2}$ be the connected components of $G-e$ with $v \in G_{1}$ and $w \in G_{2}$. Consider a path cover of $G$ consisting of minimum path covers of $G_{1}$ and $G_{2}$. Then $v$ and $w$ are not in the same path of this path cover of $G$. Also, since $\mathrm{p}_{e}(G)=0$, this path cover of $G$ is minimum.

Vertex spread. In this section, we consider the effects on minimum rank, maximum nullity, zero forcing number, and path cover number when deleting a single vertex from a graph. For a graph $G$ and a vertex $v$ of $G$, the rank spread of $v$ in $G$ is $\mathrm{r}_{v}(G)=\operatorname{mr}(G)-\operatorname{mr}(G-v)$ [Barioli et al. 2004], the null spread of $v$ in $G$ is $\mathrm{n}_{v}(G)=\mathrm{M}(G)-\mathrm{M}(G-v)$ [Edholm et al. 2010], the zero spread of $v$ in $G$ is $\mathrm{z}_{v}(G)=\mathrm{Z}(G)-\mathrm{Z}(G-v)$ [Edholm et al. 2010], and the path spread of $v$ in $G$ is $\mathrm{p}_{v}(G)=\mathrm{P}(G)-\mathrm{P}(G-v)$ [Barioli et al. 2005]. 
Theorem 2.16 [Edholm et al. 2010; Huang et al. 2010]. For every graph $G$ and vertex $v$ of $G,-1 \leq \mathrm{z}_{v}(G) \leq 1$.

Theorem 2.17 [Barioli et al. 2004; Barioli et al. 2005]. For every graph $G$ and vertex $v$ of $G,-1 \leq \mathrm{p}_{v}(G) \leq 1$.

Recall that $v$ being contained as a singleton means it is in a forcing chain by itself in an optimal chain set, and $v$ being doubly terminal means it is in a path by itself in a minimum path cover.

Theorem 2.18 [Edholm et al. 2010]. Let $v$ be a vertex of $G$. Then $\mathrm{z}_{v}(G)=1$ if and only if there exists an optimal chain set of $G$ that contains $v$ as a singleton.

Theorem 2.19 [Barioli et al. 2005]. Let $v$ be a vertex of $G$. Then $\mathrm{p}_{v}(G)=1$ if and only if $v$ is doubly terminal.

Theorem 2.20 [Edholm et al. 2010]. Let $v$ be a vertex of $G$. If $\mathrm{z}_{v}(G)=-1$, then $v$ is never in an optimal zero forcing set for $G$.

Theorem 2.21 [Barioli et al. 2005]. Let $v$ be a vertex of $G$. If $\mathrm{p}_{v}(G)=-1$, then $v$ is not terminal.

The next theorems give the parameter spreads for a cut-vertex. Recall that $v$ being simply terminal means that $v$ is terminal but not doubly terminal. By Theorems 2.19 and 2.21, this is equivalent to the path spread being zero and $v$ being an endpoint in some minimal path cover.

Theorem 2.22 [Barioli et al. 2004]. Let $G=\left(V_{G}, E_{G}\right)$ be a graph with cut-vertex $v \in V_{G}$. Let $W_{1}, \ldots, W_{k}$ be the vertex sets for the connected components of $G-v$, and for $1 \leq i \leq k$, let $G_{i}=G\left[W_{i} \cup\{v\}\right]$. Then

$$
\mathrm{r}_{v}(G)=\min \left\{\sum_{i=1}^{k} \mathrm{r}_{v}\left(G_{i}\right), 2\right\}
$$

Corollary 2.23. Let $G=\left(V_{G}, E_{G}\right)$ be a graph with cut-vertex $v \in V_{G}$. Let $W_{1}, \ldots, W_{k}$ be the vertex sets for the connected components of $G-v$, and for $1 \leq i \leq k$, let $G_{i}=G\left[W_{i} \cup\{v\}\right]$. Let $m$ denote $\min _{1 \leq j \leq k}\left\{\mathrm{n}_{v}\left(G_{j}\right)\right\}$, and $t$ denote the number of the $G_{i}$ 's in which $\mathrm{n}_{v}\left(G_{i}\right)=0$. Then

$$
\mathrm{n}_{v}(G)=\left\{\begin{aligned}
1 & \text { if } m=1 \\
0 & \text { if } m=0 \text { and } t=1, \\
-1 & \text { if } m=0 \text { and } t \geq 2, \text { or if } m=-1 .
\end{aligned}\right.
$$

Proof. This follows from Theorem 2.22 and the fact that $\mathrm{r}_{v}(G)+\mathrm{n}_{v}(G)=1$ for any graph $G$ and any vertex $v$ of $G$. 
Theorem 2.24 [Row 2011]. Let $G=\left(V_{G}, E_{G}\right)$ be a graph with cut-vertex $v \in V_{G}$. Let $W_{1}, \ldots, W_{k}$ be the vertex sets for the connected components of $G-v$, and for $1 \leq i \leq k$, let $G_{i}=G\left[W_{i} \cup\{v\}\right]$. Let $m$ denote $\min _{1 \leq j \leq k}\left\{z_{v}\left(G_{j}\right)\right\}$, and $t$ denote the number of the $G_{i}$ 's in which $\mathrm{z}_{v}\left(G_{i}\right)=0$ and $v$ is in an optimal zero forcing set. Then

$$
\mathrm{z}_{v}(G)=\left\{\begin{aligned}
1 & \text { if } m=1, \\
0 & \text { if } m=0 \text { and } t \leq 1, \\
-1 & \text { if } m=0 \text { and } t \geq 2, \text { or if } m=-1 .
\end{aligned}\right.
$$

Theorem 2.25 [Barioli et al. 2005]. Let $G=\left(V_{G}, E_{G}\right)$ be a graph with cut-vertex $v \in V_{G}$. Let $W_{1}, \ldots, W_{k}$ be the vertex sets for the connected components of $G-v$, and for $1 \leq i \leq k$, let $G_{i}=G\left[W_{i} \cup\{v\}\right]$. Let $m$ denote $\min _{1 \leq j \leq k}\left\{\mathrm{p}_{v}\left(G_{j}\right)\right\}$, and $t$ denote the number of the $G_{i}$ 's in which $v$ is simply terminal. Then

$$
\mathrm{p}_{v}(G)=\left\{\begin{aligned}
1 & \text { if } m=1, \\
0 & \text { if } m=0 \text { and } t \leq 1, \\
-1 & \text { if } m=0 \text { and } t \geq 2, \text { or if } m=-1 .
\end{aligned}\right.
$$

\section{Comparing $\mathrm{Z}(G)$ and $\mathrm{P}(G)$ for cacti}

A block of a graph is a maximal connected subgraph without a cut-vertex. A cactus is a graph in which each block is either a cycle or an edge. In other words, a cactus is a graph in which any two cycles share at most one vertex. An example of a cactus is shown in Figure 1. In this section, we prove $\mathrm{Z}(G)=\mathrm{P}(G)$ for any cactus $G$. We begin with a few preliminaries.

Theorem 3.1 [Row 2011]. Let $G$ be a unicyclic graph. Then $\mathrm{Z}(G)=\mathrm{P}(G)$.

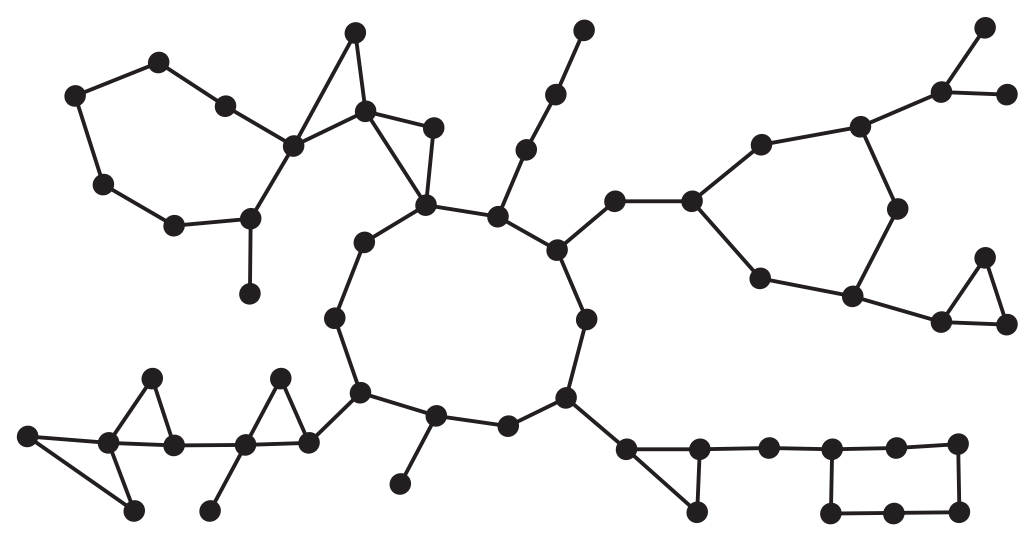

Figure 1. A cactus. No edge is in more than one cycle. 
Lemma 3.2. Let $G$ be a graph, $v$ a vertex in $G$, and $H$ the graph constructed by appending a leaf $w$ to $v$ in $G$. Suppose $\mathrm{Z}(G)=\mathrm{P}(G)$ and $\mathrm{Z}(H)=\mathrm{P}(H)$. The vertex $v$ is in an optimal zero forcing set for $G$ if and only if $v$ is terminal in $G$.

Proof. Suppose $v$ is in an optimal zero forcing set for $G$. An optimal chain set from this optimal zero forcing set determines a path cover of $G$ with $\mathrm{Z}(G)=\mathrm{P}(G)$ paths and $v$ as an endpoint of a path. Hence $v$ is terminal.

Suppose $v$ is terminal in $G$. Then $e=\{v, w\}$ is a cut edge and the graph $H^{\prime}=(\{w\}, \varnothing)$ is a single isolated vertex. Therefore, $w$ is terminal in $H^{\prime}$. By Theorem 2.13, $\mathrm{p}_{e}(H)=-1$. By Observation 2.9, $\mathrm{z}_{e}(H)=-1$. By Theorem 2.12, $v$ is in an optimal zero forcing set for $G$.

Theorem 3.3. Let $G$ be a cactus. Then $\mathrm{Z}(G)=\mathrm{P}(G)$.

Proof. The theorem will be proved by induction on the number of cycles in the cactus. If there is one cycle, $G$ is a unicyclic graph and by Theorem 3.1, $\mathrm{Z}(G)=\mathrm{P}(G)$. Suppose now that for some $m \geq 2$ any cactus $G$ with less than $m$ cycles satisfies $\mathrm{Z}(G)=\mathrm{P}(G)$. Let $G$ be a cactus with $m$ cycles. Since the cycles are edge disjoint, there is a cut-vertex $v$ such that $G-v$ has connected components with vertex sets $W_{1}, \ldots, W_{k}$ and each graph $G_{i}=G\left[W_{i} \cup\{v\}\right], \forall i=1, \ldots k$ is a cactus with fewer than $m$ cycles. By the inductive hypothesis, $\mathrm{Z}\left(G_{i}\right)=\mathrm{P}\left(G_{i}\right), \forall i=1, \ldots, k$ and $\mathrm{Z}\left(G_{i}-v\right)=\mathrm{P}\left(G_{i}-v\right), \forall i=1, \ldots, k$, so $\mathrm{z}_{v}\left(G_{i}\right)=\mathrm{p}_{v}\left(G_{i}\right), \forall i=1, \ldots, k$. Therefore, $\min _{1 \leq j \leq k}\left\{\mathrm{z}_{v}\left(G_{j}\right)\right\}=\min _{1 \leq j \leq k}\left\{\mathrm{p}_{v}\left(G_{j}\right)\right\}$. For all $i=1, \ldots k$, consider the graphs $H_{i}$ constructed by appending a leaf $w_{i}$ to $v$ in $G_{i}$. By the inductive hypothesis, $\mathrm{Z}\left(G_{i}\right)=\mathrm{P}\left(G_{i}\right), \forall i=1, \ldots k$ and $\mathrm{Z}\left(H_{i}\right)=\mathrm{P}\left(H_{i}\right), \forall i=1, \ldots k$. By Lemma 3.2, $v$ is in an optimal zero forcing set for $G_{j}$ if and only if $v$ is terminal in $G_{j}$. Then $\mathrm{z}_{v}\left(G_{j}\right)=0$ and $v$ is in an optimal zero forcing set for $G_{j}$ if and only if $\mathrm{p}_{v}\left(G_{j}\right)=0$ and $v$ is terminal in $G_{j}$ if and only if $v$ is simply terminal in $G_{j}$ by the contrapositive of Theorem 2.19. Then by Theorems 2.24 and 2.25, $\mathrm{z}_{v}(G)=\mathrm{p}_{v}(G)$. Hence $\mathrm{Z}(G)=\sum_{i=1}^{k} \mathrm{Z}\left(G_{i}-v\right)+\mathrm{z}_{v}(G)=\sum_{i=1}^{k} \mathrm{P}\left(G_{i}-v\right)+\mathrm{p}_{v}(G)=\mathrm{P}(G)$.

\section{Comparing $Z(G)$ and $M(G)$ for cacti}

In Section 3 we showed equality of $\mathrm{Z}(G)$ and $\mathrm{P}(G)$ for all cacti $G$ by utilizing Theorem 3.1 for the base case in the induction proof. Since it is not true that $\mathrm{Z}(G)=\mathrm{M}(G)$ for all unicyclic graphs, in this section we focus on a subset of cacti and prove $\mathrm{Z}(G)=\mathrm{M}(G)$ for each graph in this subset.

Let $C_{n}$ be an $n$-cycle and let $U \subseteq V_{C_{n}}$. The graph $H$ obtained from $C_{n}$ by appending a leaf to each vertex in $U$ is called a partial $n$-sun. If $U=V_{C_{n}}$, then $H$ is called an $n$-sun. It was shown in [Barioli et al. 2005] that $\mathrm{M}(H)=\mathrm{P}(H)$ for partial $n$-suns except for $n$-suns with $n>3$ odd.

If there are at least two components of the graph $G-v$ which are paths, each joined to $v$ in $G$ at only one endpoint, then vertex $v$ is called appropriate. A 
vertex $v$ is called a peripheral leaf if $v$ is adjacent to only one other vertex $u$, and $u$ is adjacent to no more than two vertices. The trimmed form of a graph $G$ is an induced subgraph obtained by a sequence of deletions of appropriate vertices, isolated paths, and peripheral leaves until no more such deletions are possible.

Theorem 4.1 [Row 2011]. If the trimmed form of $G, \breve{G}$, can be obtained by performing $n_{1}$ deletions of appropriate vertices, $n_{2}$ deletions of isolated paths, and $n_{3}$ deletions of peripheral leaves, then $\mathrm{Z}(G)=\mathrm{Z}(\breve{G})+n_{2}-n_{1}$.

Theorem 4.2 [Barioli et al. 2005]. If the trimmed form of $G, \breve{G}$, can be obtained by performing $n_{1}$ deletions of appropriate vertices, $n_{2}$ deletions of isolated paths, and $n_{3}$ deletions of peripheral leaves, then $\mathrm{M}(G)=\mathrm{M}(\breve{G})+n_{2}-n_{1}$.

Theorem 4.3 [Barioli et al. 2005]. The trimmed form of a unicyclic graph $G$ is either the empty graph or a partial n-sun.

Observation 4.4. The trimmed form of a unicyclic graph $G$ in which at least one of the cycle vertices has only two neighbors is not an $n$-sun.

The following theorem and lemma will be used in the proof of Theorem 4.7, the main result of this section.

Theorem 4.5. Let $G$ be a unicyclic graph in which the cycle has three vertices, an even number of vertices, or a vertex which has only two neighbors. Then $\mathrm{Z}(G)=$ $\mathrm{M}(G)$.

Proof. Let $\breve{G}$ be the trimmed form of $G$. By Theorem 4.3 and Observation 4.4, $\breve{G}$ is either the empty graph or a partial $n$-sun, but not an $n$-sun with $n$ odd and greater than three. The formulas from [Barioli et al. 2005] give $\mathrm{M}(\breve{G})=\mathrm{P}(\breve{G})$. Theorem 3.1 gives $\mathrm{Z}(\breve{G})=\mathrm{P}(\breve{G})$, so $\mathrm{Z}(\breve{G})=\mathrm{M}(\breve{G})$. Then $\mathrm{Z}(G)=\mathrm{M}(G)$ by Theorems 4.1 and 4.2 .

Lemma 4.6. Let $G$ be a graph, $v$ a vertex in $G$, and $H$ the graph constructed from $G$ by appending a leaf $w$ to $v$, then appending a leaf $x$ to $w$. Suppose $\mathrm{Z}(G)=\mathrm{M}(G)$ and $\mathrm{Z}(H)=\mathrm{M}(H)$. The vertex $v$ is in an optimal zero forcing set for $G$ if and only if $\mathrm{n}_{v}(G)=0$.

Proof. By construction, $e=\{v, w\}$ is a cut edge and the graph

$$
H^{\prime}=\{\{w, x\},\{\{w, x\}\}\}
$$

is a path on two vertices. Since $\mathrm{Z}\left(H^{\prime}\right)=\mathrm{M}\left(H^{\prime}\right), \mathrm{z}_{e}(H)=\mathrm{n}_{e}(H)$. Also, $w$ is in an optimal zero forcing set for $H^{\prime}$ and $n_{w}\left(H^{\prime}\right)=0$. Then $\mathrm{n}_{v}(G)=0 \Leftrightarrow \mathrm{n}_{e}(H)=$ $-1 \Leftrightarrow \mathrm{z}_{e}(H)=-1 \Leftrightarrow v$ is in an optimal zero forcing set for $G$ by Corollary 2.11 and Theorem 2.12.

Here we present the main result of the section. 
Theorem 4.7. Let $G$ be a cactus in which each cycle has three vertices, an even number of vertices, or a vertex which has only two neighbors. Then $\mathrm{Z}(G)=\mathrm{M}(G)$.

Proof. Let $G$ be a cactus in which each cycle has three vertices, an even number of vertices, or a vertex which has only two neighbors. The theorem will be proved by induction on the number of cycles in the cactus. If there is one cycle, $G$ is a unicyclic graph in which the cycle has three vertices, an even number of vertices, or a vertex which has only two neighbors, and by Theorem 4.5, $\mathrm{Z}(G)=\mathrm{M}(G)$. Suppose now that for some $m \geq 2$ any cactus $G$ in which each cycle has three vertices, an even number of vertices, or a vertex which has only two neighbors with less than $m$ cycles satisfies $\mathrm{Z}(G)=\mathrm{M}(G)$. Let $G$ be a cactus in which each cycle has three vertices, an even number of vertices, or a vertex which has only two neighbors with $m$ cycles. Since the cycles are edge disjoint, there is a cut-vertex $v$ such that $G-v$ has connected components with vertex sets $W_{1}, \ldots, W_{k}$ and each graph $G_{i}=G\left[W_{i} \cup\{v\}\right], \forall i=1, \ldots k$ is a cactus in which each cycle has three vertices, an even number of vertices, or a vertex which has only two neighbors with fewer than $m$ cycles. By the inductive hypothesis, $\mathrm{Z}\left(G_{i}\right)=\mathrm{M}\left(G_{i}\right), \forall i=1, \ldots, k$ and $\mathrm{Z}\left(G_{i}-v\right)=\mathrm{M}\left(G_{i}-v\right), \forall i=1, \ldots, k$, so $\mathrm{z}_{v}\left(G_{i}\right)=\mathrm{n}_{v}\left(G_{i}\right), \forall i=1, \ldots, k$. Therefore, $\min _{1 \leq j \leq k}\left\{\mathrm{z}_{v}\left(G_{j}\right)\right\}=\min _{1 \leq j \leq k}\left\{\mathrm{n}_{v}\left(G_{j}\right)\right\}$. For all $i=1, \ldots k$, consider the graphs $H_{i}$ constructed by appending a leaf $w_{i}$ to $v$ in $G_{i}$ then appending a leaf $x_{i}$ to $w_{i}$. By the inductive hypothesis, $\mathrm{Z}\left(G_{i}\right)=\mathrm{M}\left(G_{i}\right), \forall i=1, \ldots k$ and $\mathrm{Z}\left(H_{i}\right)=\mathrm{M}\left(H_{i}\right), \forall i=1, \ldots k$. By Lemma 4.6, $v$ is in an optimal zero forcing set for $G_{j}$ if and only if $\mathrm{n}_{v}\left(G_{j}\right)=0$. Then $\mathrm{z}_{v}\left(G_{j}\right)=0$ and $v$ is in an optimal zero forcing set for $G_{j}$ if and only if $\mathrm{n}_{v}\left(G_{j}\right)=0$. Then by Theorem 2.24 and Corollary 2.23, $\mathrm{z}_{v}(G)=\mathrm{n}_{v}(G)$. Hence

$$
\mathrm{Z}(G)=\sum_{i=1}^{k} \mathrm{Z}\left(G_{i}-v\right)+\mathrm{z}_{v}(G)=\sum_{i=1}^{k} \mathrm{M}\left(G_{i}-v\right)+\mathrm{n}_{v}(G)=\mathrm{M}(G) .
$$

The restrictions imposed on the cacti in this section are sufficient for $\mathrm{Z}(G)=$ $\mathrm{M}(G)$, but are not necessary, as can be seen in the following example.

Example 4.8. The graph $G$ shown in Figure 2 does not satisfy the property that each odd cycle of size five or more has at least one vertex with only two neighbors, but does satisfy $\mathrm{Z}(G)=\mathrm{M}(G)$.

\section{Conclusions and open questions}

We utilized cut-vertex and cut-edge results for zero forcing number, path cover number, and maximum nullity to build graphs having equality of parameters from smaller graphs having equality of the same parameters. Specifically, from knowing $\mathrm{Z}(G)=\mathrm{P}(G)$ for unicyclic graphs we showed $\mathrm{Z}(G)=\mathrm{P}(G)$ for cacti, and from 


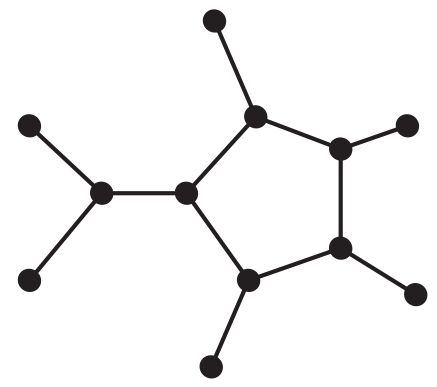

Figure 2. A cactus $G$ that is not in the restricted family but which satisfies $\mathrm{Z}(G)=\mathrm{M}(G)$.

$\mathrm{Z}(G)=\mathrm{M}(G)$ for a restricted family of unicyclic graphs we showed $\mathrm{Z}(G)=\mathrm{M}(G)$ for a restricted family of cacti.

Question 5.1. What other graphs with equality of some parameters have additional properties that would allow cut-vertex and cut-edge results to be utilized to "build" larger graphs having equality of the parameters?

Question 5.2. What are necessary conditions for a cactus to satisfy $\mathrm{Z}(G)=\mathrm{M}(G)$ ?

The converse of Theorem 2.4 is open from [Edholm et al. 2010]. We proved the converse holds if $e$ is a cut-edge. We also proved the converse of Theorem 2.5 holds for a cut-edge.

Question 5.3. Is the converse of Theorem 2.5 true? That is, if $v$ and $w$ are in the same path for every minimum path cover of $G$, does $\mathrm{p}_{e}(G)=-1$ where $e=\{v, w\}$ ?

In general, $v$ being in an optimal zero forcing set does not imply it being terminal, nor does $v$ being terminal imply it being in an optimal zero forcing set, as evidenced by Examples 5.5 and 5.6 below. With the hypothesis that $\mathrm{Z}(G)=\mathrm{P}(G)$, we do get $v$ in an optimal zero forcing set implying $v$ terminal, as can be seen in the first part of the proof for Lemma 3.2 where the graph $H$ is not used. The hypothesis about $H$ is needed in Lemma 4.6 (see Example 5.7).

Question 5.4. Is the graph $H$ from the hypothesis of Lemma 3.2 necessary for the conclusion? For a graph $G$ with $\mathrm{Z}(G)=\mathrm{P}(G)$, does vertex $v$ being terminal imply $v$ is in an optimal zero forcing set?

Example 5.5. The vertex $v$ is a cut-vertex for this graph $G$ :

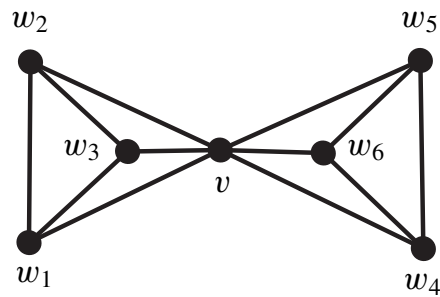


Now both $G\left[\left\{v, w_{1}, w_{2}, w_{3}\right\}\right]$ and $G\left[\left\{v, w_{4}, w_{5}, w_{6}\right\}\right]$ are $K_{4}$, so we can write $\mathrm{z}_{v}\left(G\left[\left\{v, w_{1}, w_{2}, w_{3}\right\}\right]\right)=\mathrm{z}_{v}\left(G\left[\left\{v, w_{4}, w_{5}, w_{6}\right\}\right]\right)=1$ and $v$ is simply terminal in $G\left[\left\{v, w_{1}, w_{2}, w_{3}\right\}\right]$ and $G\left[\left\{v, w_{4}, w_{5}, w_{6}\right\}\right]$. Hence $\mathrm{z}_{v}(G)=1$ and $\mathrm{p}_{v}(G)=-1$ by Theorems 2.24 and 2.25. Therefore, $v$ is in an optimal zero forcing set but not terminal by Theorems 2.18 and 2.21.

Example 5.6. Let $G$ be this graph:

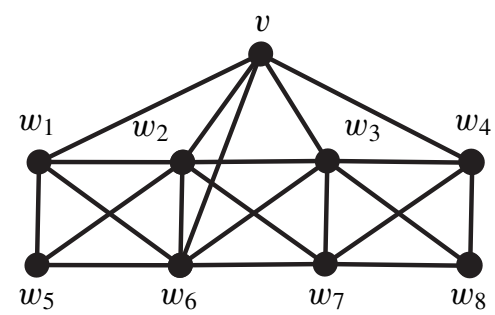

Then $\mathrm{Z}(G-v)=5$ by [AIM 2008]. By Theorem 2.16, $\mathrm{Z}(G) \geq 4$ and moreover $\left\{w_{2}, w_{3}, w_{5}, w_{6}\right\}$ is a zero forcing set, so $\mathrm{Z}(G)=4$. The graph $G-v$ is not a path, so $\mathrm{P}(G-v) \geq 2$ and $\left\{\left(w_{1}, w_{2}, w_{3}, w_{4}, w_{5}\right),\left(w_{6}, w_{7}, w_{8}, w_{9}, w_{10}\right)\right\}$ is a path cover for $G-v$. Therefore, $\mathrm{P}(G-v)=2$. By Theorem 2.17, and considering $G$ is not a path, $2 \leq \mathrm{P}(G) \leq 3$. To show $\mathrm{P}(G) \neq 2$, attempt to cover $G$ with two induced paths and consider $w_{5}$. If $w_{5}$ was in a path by itself, the other eight vertices cannot be covered with a single induced path, so $w_{5}$ has to be in a path with other vertices. Since the three neighbors of $w_{5}$ are all neighbors of each other, $w_{5}$ has to be an endpoint of an induced path. Consider which neighbor is in the path with $w_{5}$. If $w_{1}$ is with $w_{5}$, then $w_{2}$ and $w_{6}$ have to be in the other path, then $v, w_{3}$, and $w_{7}$ have to be with $w_{5}$ and $w_{1}$, then $w_{4}$ and $w_{8}$ have to be with $w_{2}$ and $w_{6}$, but $G\left[\left\{w_{2}, w_{4}, w_{6}, w_{8}\right\}\right]$ is not a path. If $w_{2}$ is with $w_{5}$, then $w_{1}$ and $w_{6}$ have to be in the other path, then $v$ has to be with $w_{5}$ and $w_{2}$, then $w_{3}$ has to be with $w_{1}$ and $w_{6}$, then $w_{7}$ has to be with $w_{5}$, $w_{2}$, and $v$, but $G\left[\left\{v, w_{2}, w_{5}, w_{7}\right\}\right]$ is not a path. If $w_{6}$ is with $w_{5}$, then $w_{1}$ and $w_{2}$ have to be in the other path, then $v$ has to be with $w_{5}$ and $w_{6}$, then $w_{3}$ has to be with $w_{1}$ and $w_{2}$, then $w_{7}$ has to be with $w_{5}, w_{6}$, and $v$, but $G\left[\left\{v, w_{5}, w_{6}, w_{7}\right\}\right]$ is not a path. So $\mathrm{P}(G) \geq 3$. Hence $\mathrm{z}_{v}(G)=-1$ and $\mathrm{p}_{v}(G)=1$. Hence, $v$ is terminal but never in an optimal zero forcing set by Theorems 2.19 and 2.20.

Example 5.7. Let $G$ be this graph:

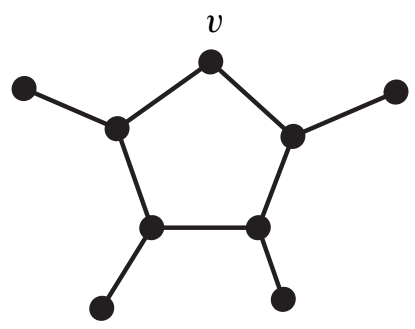


Then $\mathrm{Z}(G)=\mathrm{M}(G)$ and $\mathrm{n}_{v}(G)=0$, but $v$ is not in an optimal zero forcing set for $G$.

\section{References}

[AIM 2008] AIM Minimum Rank - Special Graphs Work Group (F. Barioli, W. Barrett, S. Butler, S. M. Cioabă, D. Cvetković, S. M. Fallat, C. Godsil, W. Haemers, L. Hogben, R. Mikkelson, S. Narayan, O. Pryporova, I. Sciriha, W. So, D. Stevanović, H. van der Holst, K. Vander Meulen, A. W. Wehe), "Zero forcing sets and the minimum rank of graphs", Linear Algebra Appl. 428:7 (2008), 1628-1648. MR 2008m:05166 Zbl 1135.05035

[Barioli et al. 2004] F. Barioli, S. Fallat, and L. Hogben, "Computation of minimal rank and path cover number for certain graphs", Linear Algebra Appl. 392 (2004), 289-303. MR 2005i:05115 Zbl 1052.05045

[Barioli et al. 2005] F. Barioli, S. Fallat, and L. Hogben, "On the difference between the maximum multiplicity and path cover number for tree-like graphs", Linear Algebra Appl. 409 (2005), 13-31. MR 2006e:05038 Zbl 1072.05037

[Barioli et al. 2009] F. Barioli, S. M. Fallat, H. T. Hall, D. Hershkowitz, L. Hogben, H. van der Holst, and B. Shader, "On the minimum rank of not necessarily symmetric matrices: a preliminary study”, Electron. J. Linear Algebra 18 (2009), 126-145. MR 2010e:05176 Zbl 1169.05345

[Barioli et al. 2010] F. Barioli, W. Barrett, S. M. Fallat, H. T. Hall, L. Hogben, B. Shader, P. van den Driessche, and H. van der Holst, "Zero forcing parameters and minimum rank problems", Linear Algebra Appl. 433:2 (2010), 401-411. MR 2011g:15002 Zbl 1209.05139

[Burgarth and Giovannetti 2007] D. Burgarth and V. Giovannetti, "Full control by locally induced relaxation", Phys. Rev. Lett. 99 (2007), Art. ID 100501.

[Burgarth and Maruyama 2009] D. Burgarth and K. Maruyama, "Indirect Hamiltonian identification through a small gateway", 2009. arXiv 0903.0612

[Edholm et al. 2010] C. J. Edholm, L. Hogben, M. Huynh, J. LaGrange, and D. D. Row, "Vertex and edge spread of zero forcing number, maximum nullity, and minimum rank of a graph", Linear Algebra Appl., available online 18 November 2010.

[Hogben 2010] L. Hogben, "Minimum rank problems", Linear Algebra Appl. 432:8 (2010), 19611974. MR 2011b:15002 Zbl 1213.05036

[Huang et al. 2010] L.-H. Huang, G. J. Chang, and H.-G. Yeh, "On minimum rank and zero forcing sets of a graph”, Linear Algebra Appl. 432 (2010), 2961-2973. MR 2011g:05183 Zbl 1195.05043

[Johnson and Duarte 1999] C. R. Johnson and A. L. Duarte, "The maximum multiplicity of an eigenvalue in a matrix whose graph is a tree: invariant factors", Linear Algebra Appl. 46:1-2 (1999), 139-144. MR 2000e:05114 Zbl 0929.15005

[Row 2011] D. D. Row, "A technique for computing the zero forcing number of a graph with a cut-vertex", Linear Algebra Appl., available online 21 June 2011.

[Severini 2008] S. Severini, "Nondiscriminatory propagation on trees", J. Phys. A 41:48 (2008), Art. ID 482002. MR 2010h:82067

Received: 2011-05-05 Revised: 2011-05-12 Accepted: 2011-05-13

rowd@uiu.edu

Division of Science and Mathematics, Upper lowa University, Fayette, IA 52142, United States 
EDITORS

MANAGING EDITOR

Kenneth S. Berenhaut, Wake Forest University, USA, berenhks@wfu.edu

\section{BOARD OF EDITORS}

John V. Baxley Wake Forest University, NC, USA baxley@wfu.edu

Arthur T. Benjamin Harvey Mudd College, USA

benjamin@hmc.edu

Martin Bohner Missouri U of Science and Technology, USA bohner@mst.edu

Nigel Boston University of Wisconsin, USA boston@math.wisc.edu

Amarjit S. Budhiraja $\quad$ U of North Carolina, Chapel Hill, USA budhiraj@email.unc.edu

Pietro Cerone Victoria University, Australia pietro.cerone@vu.edu.au

Scott Chapman Sam Houston State University, USA scott.chapman@shsu.edu

Jem N. Corcoran University of Colorado, USA corcoran@colorado.edu

Toka Diagana Howard University, USA tdiagana@howard.edu

Michael Dorff Brigham Young University, USA mdorff@math.byu.edu

Sever S. Dragomir Victoria University, Australia sever@matilda.vu.edu.au

Behrouz Emamizadeh

Errin W. Fulp

Joseph Gallian

Stephan R. Garcia

Ron Gould

Andrew Granville

Jerrold Griggs

Ron Gould

Sat Gupta

The Petroleum Institute, UAE

bemamizadeh@pi.ac.ae

Wake Forest University, USA fulp@wfu.edu

University of Minnesota Duluth, USA

jgallian@d.umn.edu

Pomona College, USA

stephan.garcia@pomona.edu

Emory University, USA

rg@mathcs.emory.edu

Université Montréal, Canada andrew@dms.umontreal.ca

University of South Carolina, USA

griggs@math.sc.edu

Emory University, USA

rg@mathcs.emory.edu

Jim Haglund $\quad \begin{aligned} & \text { University of Pennsylvania, USA } \\ & \text { sngupta@uncg.edu }\end{aligned}$

jhaglund@math.upenn.edu

Johnny Henderson Baylor University, USA

johnny_henderson@baylor.edu

Natalia Hritonenko Prairie View A\&M University, USA nahritonenko@pvamu.edu

Charles R. Johnson College of William and Mary, USA crjohnso@math.wm.edu

Karen Kafadar University of Colorado, USA

karen.kafadar@cudenver.edu

K. B. Kulasekera Clemson University, USA

kk@ces.clemson.edu

Gerry Ladas University of Rhode Island, USA gladas@math.uri.edu

David Larson Texas A\&M University, USA larson@math.tamu.edu

Suzanne Lenhart University of Tennessee, USA lenhart@math.utk.edu
Chi-Kwong Li

Robert B. Lund

Gaven J. Martin

Mary Meyer

Emil Minchev

Frank Morgan

Mohammad Sal Moslehian

Zuhair Nashed

Ken Ono

Timothy E. O'Brien

Joseph O'Rourke

Yuval Peres

Y.-F. S. Pétermann

Robert J. Plemmons

Carl B. Pomerance

Vadim Ponomarenko

Bjorn Poonen

James Propp

Józeph H. Przytycki

Richard Rebarber

Robert W. Robinson

Filip Saidak

James A. Sellers

Andrew J. Sterge

Ann Trenk

Ravi Vakil

Ram U. Verma

John C. Wierman

Michael E. Zieve
College of William and Mary, USA ckli@math.wm.edu

Clemson University, USA

lund@clemson.edu

Massey University, New Zealand

g.j.martin@massey.ac.nz

Colorado State University, USA

meyer@stat.colostate.edu

Ruse, Bulgaria

eminchev@ hotmail.com

Williams College, USA

frank.morgan@williams.edu

Ferdowsi University of Mashhad, Iran moslehian@ferdowsi.um.ac.ir

University of Central Florida, USA

znashed@mail.ucf.edu

Emory University, USA

ono@mathcs.emory.edu

Loyola University Chicago, USA

tobrie1@luc.edu

Smith College, USA

orourke@cs.smith.edu

Microsoft Research, USA

peres@microsoft.com

Université de Genève, Switzerland petermann@math.unige.ch

Wake Forest University, USA plemmons@wfu.edu

Dartmouth College, USA

carl.pomerance@dartmouth.edu

San Diego State University, USA

vadim@sciences.sdsu.edu

UC Berkeley, USA

poonen@math.berkeley.edu

U Mass Lowell, USA

jpropp@cs.uml.edu

George Washington University, USA

przytyck@gwu.edu

University of Nebraska, USA

rrebarbe@math.unl.edu

University of Georgia, USA

rwr@cs.uga.edu

U of North Carolina, Greensboro, USA

f_saidak@uncg.edu

Penn State University, USA

sellersj@math.psu.edu

Honorary Editor

andy@ajsterge.com

Wellesley College, USA

atrenk@wellesley.edu

Stanford University, USA

vakil@math.stanford.edu

University of Toledo, USA

verma99@msn.com

Johns Hopkins University, USA

wierman@jhu.edu

University of Michigan, USA

zieve@umich.edu

\section{PRODUCTION}

Silvio Levy, Scientific Editor

Sheila Newbery, Senior Production Editor

Cover design: (C2008 Alex Scorpan

See inside back cover or http://msp.berkeley.edu/involve for submission instructions.

The subscription price for 2011 is US \$100/year for the electronic version, and \$130/year (+\$35 shipping outside the US) for print and electronic. Subscriptions, requests for back issues from the last three years and changes of subscribers address should be sent to Mathematical Sciences Publishers, Department of Mathematics, University of California, Berkeley, CA 94704-3840, USA.

Involve (ISSN 1944-4184 electronic, 1944-4176 printed) at Mathematical Sciences Publishers, Department of Mathematics, University of California, Berkeley, CA 94720-3840 is published continuously online. Periodical rate postage paid at Berkeley, CA 94704, and additional mailing offices.

Involve peer review and production are managed by EditFLOW ${ }^{\mathrm{TM}}$ from Mathematical Sciences Publishers.

PUBLISHED BY

mathematical sciences publishers

http://msp.org/

A NON-PROFIT CORPORATION

Typeset in IATEX

Copyright $@ 2011$ by Mathematical Sciences Publishers 


\section{involve 2011 vol. $4 \quad$ no. 3}

An implementation of scatter search to train neural networks for brain lesion recognition JEFFREY LARSON AND FRANCIS NEWMAN

$\mathrm{P}_{1}$ subalgebras of $M_{n}(\mathbb{C})$

StePhen Rowe, Junsheng FAng AND DAVID R. LARson

On three questions concerning groups with perfect order subsets

LENNY JONES AND KELLY TOPPIN

On the associated primes of the third power of the cover ideal

Kim Kesting, James Pozzi and JAnet StRiUli

Soap film realization of isoperimetric surfaces with boundary

Jacob Ross, Donald Sampson and NeIl Steinburg

Zero forcing number, path cover number, and maximum nullity of cacti

DARREN D. ROW

Jacobson's refinement of Engel's theorem for Leibniz algebras

Lindsey Bosko, Allison Hedges, John T. Hird, Nathaniel Schwartz And KRISTEN STAGG

The rank gradient and the lamplighter group

DEREK J. Allums AND RostislaV I. GRIGORCHUK 